\title{
A fourth order phase-fitted Runge-Kutta-Nyström method for oscillatory problems
}

\begin{abstract}
In this paper, a new Runge-Kutta-Nyström (RKN) method of fourth algebraic order is constructed. The new method is based on Dormand's Runge-Kutta-Nyström method of algebraic order four, by using the idea of phase-lag of order infinity, a new method for solving second-order ordinary differential equations (ODE) with oscillatory solutions is derived. Numerical tests show that the new method is more accurate than other existing methods that based on the minimal phase-lag theory.
\end{abstract}

Keyword: Oscillatory solutions; Phase-fitted; Phase-lag; Runge-Kutta-Nyström 